Bull. Chem. Soc. Ethiop. 2021, 35(1), 185-196.

ISSN 1011-3924

(C) 2021 Chemical Society of Ethiopia and The Authors

Printed in Ethiopia

DOI: https://dx.doi.org/10.4314/bcse.v35i1.16

Online ISSN 1726-801X

\title{
COPPER-BASED GLASS-CERAMIC AS AN EFFICIENT CATALYST IN THE SYNTHESIS OF PYRAZOLO[1,5-a]PYRIMIDINEUNDER SOLVENT-FREE CONDITION WITH DOCKING VALIDATION AS COVID-19 MAIN PROTEASE $\left(\mathrm{M}^{\mathrm{PRO}}\right)$ INHIBITOR
}

\author{
Amr M. Abdelghany ${ }^{1}$, Tamer K. Khatab ${ }^{2}$ and Ashraf S. Hassan ${ }^{2 *}$ \\ ${ }^{1}$ Spectroscopy Department, Physics Division, National Research Centre, 33 El-Behouth St., \\ Dokki, 12622, Cairo, Egypt \\ ${ }^{2}$ Organometallic and Organometalloid Chemistry Department, National Research Centre, \\ Dokki, 12622, Cairo, Egypt
}

(Received December 13, 2020; Revised March 13, 2021; Accepted March 17, 2021)

\begin{abstract}
Copper-based oxide glass-ceramic was successfully synthesized through the single-step melt annealing technique. Synthesized glass-ceramics was characterized using X-ray diffraction (XRD) and scanning electron microscopy (SEM) supported with energy dispersive X-ray (EDX) and mapping. Pyrazolo[1,5a]pyrimidines 5a-f were synthesized via the reaction of 5-amino- $1 H$-pyrazole-4-carboxamide (1) with enaminones 2a-f in the presence of synthesized oxide glass-ceramic catalyst powder under solvent-free condition. The molecular docking study demonstrated that the COVID-19 main protease $\left(\mathrm{M}^{\mathrm{Pro}}\right)$ inhibitor.
\end{abstract}

KEY WORDS: Pyrazolopyrimidine, Enaminones, Copper-based catalysis, Solvent-free, COVID-19

\section{INTRODUCTION}

During the last decades, several authors devoted their efforts to increasing the yield of specific known reactions or to reduce the time of reactions through the usage of a new catalyst [1] or to modify already used catalyst and/or converting already used one to their nanoscale [2].

Copper-based catalysts proved to have good activity for the water-gas shift reactions with no methanation activity so they are well suited for use in water-gas shift duties. However, because copper catalysts are particularly pruned to easy sintering, they can only be used with low temperatures operations [3].

Catalysts prepared from mixed oxides of copper and zinc were being demonstrated, but the problem of producing catalyst with sufficiently long life to become a commercial proposition was not resolved [4], therefore additives of aluminum oxide and silicon dioxide were added to act as a glass network matrix that holds constituents via covalent bonding between constituents [5].

Pyrazolo[1,5-a]pyrimidines have diverse biological activities [6-12] such as compound $\mathbf{A}$, pyrazolo[1,5- $a$ ]pyrimidine benzoic acid derivative, act as an antimicrobial agent [13]. Compound $\mathbf{B}, N^{1}$-(2,5-dimethyl-3- $p$-tolylpyrazolo[1,5-a]pyrimidin-7-yl)-1,3-diamine derivative, act as a potent hepatitis C virus inhibitor [14]. Compound C, 4-(6-(4-(1-(pyrrolidin-1yl)ethyl)phenyl)pyrazolo[1,5-a]pyrimidin-3-yl)naphthalene-1-sulfonamide, act as a potent and selective ALK2 inhibitor [15]. Recently, Attia et al. have synthesized the two derivatives, 7-(4methoxyphenyl)-pyrazolo[1,5-a]pyrimidine-3,6-dicarbonitrile (D) and 7-(naphthalen-2-yl)pyrazolo[1,5-a]pyrimidine-3-carbonitrile $(\mathbf{E})$, which have a potent anti-proliferative activity [16]. Also, Singleton et al. have synthesized 3-(1H-benzo[d]imidazol-2-yl)pyrazolo[1,5a]pyrimidine (F) which act as a selective inhibitor of JAK1 JH2 pseudokinase [17].

*Corresponding author. E-mail: ashraf_salmoon@yahoo.com

This work is licensed under the Creative Commons Attribution 4.0 International License 
Furthermore, some structures of drugs of pyrazolo[1,5-a]pyrimidine such as Zaleplon is a drug used to treat insomnia; Dinaciclib (SCH-727965) is a cyclin-dependent kinases inhibitor drug. Also, Ocinaplon is an anxiolytic drug.
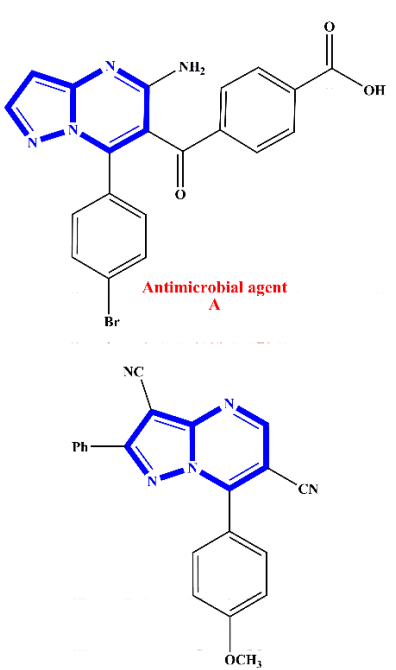

A potent anti-proliferative activity

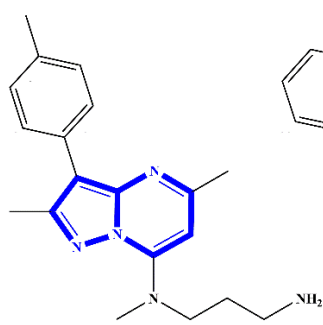

Hepatitis $\underset{B}{C}$ virus inhibitor

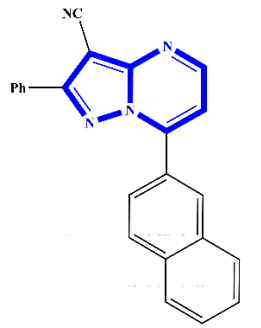

A potent anti-proliferative activity
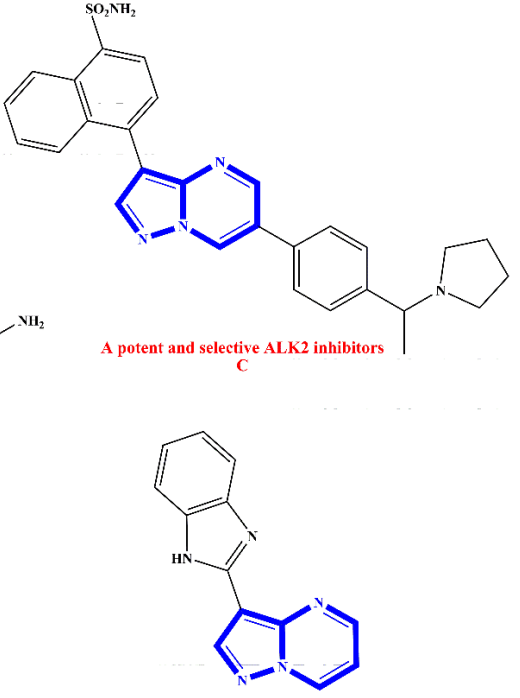

A sclective inhibitor of JAK1 JH2 pscudokinase

Figure 1. Some importantpyrazolo[1,5-a]pyrimidines (A-F).

From the above facts and in continuation of our targets [18-33], the presented work aims to introduce a new efficient copper-based oxide glass-ceramic as a catalyst in the synthesis of pyrazolo[1,5- $a]$ pyrimidines 5a-f under solvent-free condition. The work extended to study with docking validation as COVID-19 main protease $\left(\mathrm{M}^{\mathrm{pro}}\right)$ inhibitor.

\section{EXPERIMENTAL}

\section{Catalyst preparation and characterization}

Copper-based oxide glass-ceramic catalyst was prepared via a simple melt annealing technique. Pre-calculated amounts of analytical grade chemical of copper oxide, aluminum oxide, zinc oxide, and silicon dioxide were used for the synthesis of the glass-ceramic catalyst of nominal composition $30 \mathrm{SiO}_{2}-35 \mathrm{ZnO}-25 \mathrm{CuO}-10 \mathrm{Al}_{2} \mathrm{O}_{3} \mathrm{~mol} \%$. Mixed powder melted in a muffle furnace in a platinum crucible at a temperature $1350 \pm 20{ }^{\circ} \mathrm{C}$. The melt was swirled at a constant time interval to ensure homogeneity and bubble-free samples. The obtained melt was poured over a preheated stainless steel mold and transferred immediately to the annealing oven. The oven was then turned off and allowed to cool to room temperature with a cooling rate of $20^{\circ} \mathrm{C} / \mathrm{h}$.

Synthesized glass-ceramic was analyzed via XRD to identify possible present crystalline phases that precipitated within the sample during annealing process. Obtained sample was ground and the fine powder was examined using a Philips PW 1390 X-ray diffractometer 
adopting Ni-filter and $\mathrm{Cu}$-target operating at $30 \mathrm{kV}$. Obtained pattern recognized with an iterative comparison with standard JCPDS, ASTM cards, and published data.

Scanning electron microscopic (SEM) study carried out on powdered sample coated with a thin layer of gold using JSM-7500F field emission microscope supported with EDAX unit using accelerating voltage $30 \mathrm{kV}$, magnification up to $\times 400,000$.

\section{Preparation of pyrazolo[1,5-a]pyrimidines $\mathbf{5 a - f}$}

One mole of 5-amino-3-(4-methoxyphenylamino)- $N$-phenyl- $1 H$-pyrazole-4-carboxamide (1) was added to one mole of enaminones 2 a-f in the presence of catalytic amount $(10 \mathrm{wt} \%)$ of the prepared oxide glass-ceramic powder under solvent-free condition at $60-70{ }^{\circ} \mathrm{C}$, after the appropriate time the reaction was worked up using water and the organic compounds were extracted using ethyl acetate. After the separation and drying process the product was characterized as 7-aryl-2-(arylamino)pyrazolo[1,5- $a$ ]pyrimidine-3-carboxamides 5a-f [34].

2-(4-Methoxyphenylamino)-N,7-diphenylpyrazolo[1,5-a]pyrimidine-3-carboxamide(5a). Yellow crystals, m.p. $218-220{ }^{\circ} \mathrm{C}$, yield $(72 \%)$. IR $(\mathrm{KBr}) v_{\max } / \mathrm{cm}^{-1} 3346(\mathrm{NH}), 1658(\mathrm{C}=\mathrm{O}) .{ }^{1} \mathrm{H}-\mathrm{NMR}$ $\left(\mathrm{CDCl}_{3}, 400 \mathrm{MHz}, \delta \mathrm{ppm}\right): 3.80\left(\mathrm{~s}, 3 \mathrm{H}, \mathrm{OCH}_{3}\right), 6.88(\mathrm{~d}, 2 \mathrm{H}, J=9.0 \mathrm{~Hz}, \mathrm{ArH}), 6.96(\mathrm{~d}, 1 \mathrm{H}, J=$ $4.8 \mathrm{~Hz}$, pyrimidine), $7.12(\mathrm{t}, 1 \mathrm{H}, \mathrm{ArH}), 7.36-7.42(\mathrm{~m}, 5 \mathrm{H}, \mathrm{ArH}), 7.62(\mathrm{~d}, 2 \mathrm{H}, J=9.0 \mathrm{~Hz}, \mathrm{ArH})$, $7.74(\mathrm{~d}, 2 \mathrm{H}, J=8.4 \mathrm{~Hz}, \operatorname{ArH}), 8.11(\mathrm{~d}, 2 \mathrm{H}, J=8.3 \mathrm{~Hz}, \operatorname{ArH}), 8.49(\mathrm{~d}, 1 \mathrm{H}, J=4.8 \mathrm{~Hz}$, pyrimidine $), 9.40(\mathrm{~s}, 1 \mathrm{H}, \mathrm{NH}), 10.05(\mathrm{~s}, 1 \mathrm{H}, \mathrm{NH}) .{ }^{13} \mathrm{C}-\mathrm{NMR}\left(\mathrm{CDCl}_{3}, 100 \mathrm{MHz}, \delta \mathrm{ppm}\right): 55.7(\mathrm{C}$, $\left.\mathrm{OCH}_{3}\right), 87.8$ ( $\mathrm{C}, \mathrm{C}_{3}$-pyrazolopyrimidine), 107.0 (C, $\mathrm{C}_{6}$-pyrazolopyrimidine), 114.4, 119.2, 120.2, 123.7, 127.7, 129.1, 129.5, 129.6 (14C, Ar), 134.1 (C, $\mathrm{C}_{3 \mathrm{a}}$-pyrazolopyrimidine), 138.8, 142.4, 146.7 (3C, Ar), 147.9 (C, C 7 -pyrazolopyrimidine), $149.6(\mathrm{C}, \mathrm{Ar}), 154.5\left(\mathrm{C}, \mathrm{C}_{2^{-}}\right.$ pyrazolopyrimidine), $157.8\left(\mathrm{C}, \mathrm{C}_{5}\right.$-pyrazolopyrimidine), $163.3(\mathrm{C}=\mathrm{O})$. MS $(\mathrm{m} / \mathrm{z}, \%): 435\left(\mathrm{M}^{+}\right.$, 73.86). Anal. calcd. (\%) for $\mathrm{C}_{26} \mathrm{H}_{21} \mathrm{~N}_{5} \mathrm{O}_{2}$ (435.48): C, 71.71; H, 4.86; N, 16.08. Found: $\mathrm{C}, 71.80$; $\mathrm{H}, 4.81 ; \mathrm{N}, 16.00 \%$.

2-(4-Methoxyphenylamino)-N-phenyl-7-(4-methylphenyl)-pyrazolo[1,5-a]pyrimidine-3-

carboxamide (5b). Yellow crystals, m.p. 219-221 ${ }^{\circ} \mathrm{C}$, yield $(77 \%)$. IR $(\mathrm{KBr}) v_{\max } / \mathrm{cm}^{-1} 3337$ $(\mathrm{NH}), 1658(\mathrm{C}=\mathrm{O}) .{ }^{1} \mathrm{H}-\mathrm{NMR}\left(\mathrm{CDCl}_{3}, 400 \mathrm{MHz}, \delta \mathrm{ppm}\right): 2.49\left(\mathrm{~s}, 3 \mathrm{H}, \mathrm{CH}_{3}\right), 3.80(\mathrm{~s}, 3 \mathrm{H}$, $\left.\mathrm{OCH}_{3}\right), 6.87(\mathrm{~d}, 2 \mathrm{H}, J=8.9 \mathrm{~Hz}, \mathrm{ArH}), 6.91(\mathrm{~d}, 1 \mathrm{H}, J=4.7 \mathrm{~Hz}$, pyrimidine), $7.12(\mathrm{t}, 1 \mathrm{H}, \mathrm{ArH})$, 7.36 (d, 2H, $J=8.3 \mathrm{~Hz}, \mathrm{ArH}), 7.38(\mathrm{t}, 2 \mathrm{H}, \mathrm{ArH}), 7.60$ (d, 2H, $J=8.9 \mathrm{~Hz}, \operatorname{ArH}), 7.73$ (d, 2H, $J=$ $7.6 \mathrm{~Hz}, \mathrm{ArH}), 8.08(\mathrm{~d}, 2 \mathrm{H}, J=8.1 \mathrm{~Hz}, \mathrm{ArH}), 8.43(\mathrm{~d}, 1 \mathrm{H}, J=4.7 \mathrm{~Hz}$, pyrimidine $), 9.38(\mathrm{~s}, 1 \mathrm{H}$, $\mathrm{NH}), 10.01(\mathrm{~s}, 1 \mathrm{H}, \mathrm{NH}) .{ }^{13} \mathrm{C}-\mathrm{NMR}\left(\mathrm{CDCl}_{3}, 100 \mathrm{MHz}, \delta \mathrm{ppm}\right): 21.8\left(\mathrm{C}, \mathrm{CH}_{3}\right), 55.7\left(\mathrm{C}, \mathrm{OCH}_{3}\right)$, 87.7 (C, $\mathrm{C}_{3}$-pyrazolopyrimidine), 107.0 (C, $\mathrm{C}_{6}$-pyrazolopyrimidine), 114.4, 119.1, 120.1, 123.7, 127.6, 129.1, 129.4, 129.6 (14C, Ar), 134.1 (C, C ${ }_{3 \mathrm{a}}$-pyrazolopyrimidine), 138.8, 142.3, 146.6 (3C, Ar), 147.8 (C, $\mathrm{C}_{7}$-pyrazolopyrimidine), 149.6 (C, Ar), 154.4 (C, $\mathrm{C}_{2}$-pyrazolopyrimidine), 157.7 ( $\mathrm{C}, \mathrm{C}_{5}$-pyrazolopyrimidine), $163.3(\mathrm{C}=\mathrm{O})$. $\mathrm{MS}(\mathrm{m} / \mathrm{z}, \%): 449\left(\mathrm{M}^{+}, 67.43\right)$. Anal. calcd. (\%) for $\mathrm{C}_{27} \mathrm{H}_{23} \mathrm{~N}_{5} \mathrm{O}_{2}$ (449.50): C, 72.14; H, 5.16; N, 15.58. Found: C, 72.10; H, 5.20; N, 15.60\%.

7-(4-Methoxyphenyl)-2-(4-methoxyphenylamino)- $N$-phenylpyrazolo[1,5-a]pyrimidine-3-

carboxamide $(\mathbf{5 c})$. Yellow crystals, m.p. $206-208{ }^{\circ} \mathrm{C}$, yield $(76 \%)$. IR $(\mathrm{KBr}) v_{\max } / \mathrm{cm}^{-1} 3340$ $(\mathrm{NH}), 1646(\mathrm{C}=\mathrm{O}) .{ }^{1} \mathrm{H}-\mathrm{NMR}\left(\mathrm{CDCl}_{3}, 400 \mathrm{MHz}, \delta \mathrm{ppm}\right): 3.80\left(\mathrm{~s}, 3 \mathrm{H}, \mathrm{OCH}_{3}\right), 3.91(\mathrm{~s}, 3 \mathrm{H}$, $\left.\mathrm{OCH}_{3}\right), 6.87(\mathrm{~d}, 2 \mathrm{H}, J=8.9 \mathrm{~Hz}, \mathrm{ArH}), 6.89(\mathrm{~d}, 1 \mathrm{H}, J=4.8 \mathrm{~Hz}$, pyrimidine), $7.05(\mathrm{~d}, 2 \mathrm{H}, J=8.8$ $\mathrm{Hz}, \mathrm{ArH}), 7.11(\mathrm{t}, 1 \mathrm{H}, \mathrm{ArH}), 7.37$ (t, 2H, ArH), 7.60 (d, 2H, $J=8.9 \mathrm{~Hz}, \mathrm{ArH}), 7.72$ (d, 2H, $J=$ $7.6 \mathrm{~Hz}, \mathrm{ArH}), 8.18$ (d, $2 \mathrm{H}, J=8.8 \mathrm{~Hz}, \mathrm{ArH}), 8.40(\mathrm{~d}, 1 \mathrm{H}, J=4.8 \mathrm{~Hz}$, pyrimidine), $9.36(\mathrm{~s}, 1 \mathrm{H}$, $\mathrm{NH}), 10.02(\mathrm{~s}, 1 \mathrm{H}, \mathrm{NH}) .{ }^{13} \mathrm{C}-\mathrm{NMR}\left(\mathrm{CDCl}_{3}, 100 \mathrm{MHz}, \delta \mathrm{ppm}\right): 55.5\left(\mathrm{C}, \mathrm{OCH}_{3}\right), 55.6\left(\mathrm{C}, \mathrm{OCH}_{3}\right)$, 87.4 (C, $\mathrm{C}_{3}$-pyrazolopyrimidine), 106.4 (C, $\mathrm{C}_{6}$-pyrazolopyrimidine), 113.9, 114.2, 119.0, 120.0, 122.4, 123.5, 128.9, 131.3 (14C, Ar), 134.0 (C, $\mathrm{C}_{3 \mathrm{a}}$-pyrazolopyrimidine), 138.6, 146.0 (2C, Ar), 147.7 (C, $\mathrm{C}_{7}$-pyrazolopyrimidine), 149.3 (C, Ar), 154.3 (C, $\mathrm{C}_{2}$-pyrazolopyrimidine), 157.5 (C, 
$\mathrm{C}_{5}$-pyrazolopyrimidine), $162.2(\mathrm{C}, \mathrm{Ar}), 163.2(\mathrm{C}=\mathrm{O})$. $\mathrm{MS}(\mathrm{m} / \mathrm{z}, \%): 465\left(\mathrm{M}^{+}, 69.48\right)$. Anal. calcd. (\%) for $\mathrm{C}_{27} \mathrm{H}_{23} \mathrm{~N}_{5} \mathrm{O}_{3}$ (465.50): C, 69.66; H, 4.98; N, 15.04. Found: C, 69.70; H, 4.95; N, $15.00 \%$.

7-(4-Chlorophenyl)-2-(4-methoxyphenylamino)-N-phenylpyrazolo[1,5-a]pyrimidine-3-

carboxamide $(\mathbf{5 d})$. Yellow crystals, m.p. $252-253{ }^{\circ} \mathrm{C}$, yield $(72 \%)$. IR $(\mathrm{KBr}) v_{\max } / \mathrm{cm}^{-1} 3343$ $(\mathrm{NH}), 1648(\mathrm{C}=\mathrm{O}) .{ }^{1} \mathrm{H}-\mathrm{NMR}\left(\mathrm{CDCl}_{3}, 400 \mathrm{MHz}, \delta \mathrm{ppm}\right): 3.81\left(\mathrm{~s}, 3 \mathrm{H}, \mathrm{OCH}_{3}\right), 6.88(\mathrm{~d}, 2 \mathrm{H}, J=$ $9.0 \mathrm{~Hz}, \mathrm{ArH}), 6.94$ (d, 1H, J=4.7 Hz, pyrimidine), 7.13 (t, 1H, ArH), 7.39 (t, 2H, ArH), 7.58 (d, $4 \mathrm{H}, J=8.8 \mathrm{~Hz}, \mathrm{ArH}), 7.74(\mathrm{~d}, 2 \mathrm{H}, J=8.6 \mathrm{~Hz}, \mathrm{ArH}), 8.15(\mathrm{~d}, 2 \mathrm{H}, J=8.7 \mathrm{~Hz}, \mathrm{ArH}), 8.52(\mathrm{~d}, 1 \mathrm{H}$, $J=4.7 \mathrm{~Hz}$, pyrimidine), $9.42(\mathrm{~s}, 1 \mathrm{H}, \mathrm{NH}), 9.99(\mathrm{~s}, 1 \mathrm{H}, \mathrm{NH}) .{ }^{13} \mathrm{C}-\mathrm{NMR}\left(\mathrm{CDCl}_{3}, 100 \mathrm{MHz}, \delta\right.$ ppm): $55.7\left(\mathrm{C}, \mathrm{OCH}_{3}\right), 88.0\left(\mathrm{C}, \mathrm{C}_{3}\right.$-pyrazolopyrimidine $)$, $107.0\left(\mathrm{C}, \mathrm{C}_{6}\right.$-pyrazolopyrimidine $)$, $114.4, \quad 119.2, \quad 120.2,123.8,129.1,129.1,130.9,131.8 \quad(14 \mathrm{C}, \quad \mathrm{Ar}), 133.9\left(\mathrm{C}_{2} \mathrm{C}_{3 \mathrm{a}^{-}}\right.$ pyrazolopyrimidine), 134.6, 138.0, 138.7 (3C, Ar), 145.3 (C, $\mathrm{C}_{7}$-pyrazolopyrimidine), 149.7 (C, Ar), $154.6\left(\mathrm{C}, \mathrm{C}_{2}\right.$-pyrazolopyrimidine), 157.9 ( $\mathrm{C}, \mathrm{C}_{5}$-pyrazolopyrimidine), $163.2(\mathrm{C}=\mathrm{O})$. $\mathrm{MS}$ $(\mathrm{m} / \mathrm{z}, \%): 469\left(\mathrm{M}^{+}, 78.23\right)$. Anal. calcd. $(\%)$ for $\mathrm{C}_{26} \mathrm{H}_{20} \mathrm{ClN}_{5} \mathrm{O}_{2}$ (469.92): C, 66.45; H, 4.29; N, 14.90. Found: C, 66.40; H, 4.30; N, 14.95\%.

7-(4-Bromophenyl)-2-(4-methoxyphenylamino)-N-phenylpyrazolo[1,5-a]pyrimidine-3-

carboxamide (5e). Yellow crystals, m.p. $278-280{ }^{\circ} \mathrm{C}$, yield $(69 \%)$. IR $(\mathrm{KBr}) v_{\max } / \mathrm{cm}^{-1} 3365$ $(\mathrm{NH}), 1650(\mathrm{C}=\mathrm{O}) .{ }^{1} \mathrm{H}-\mathrm{NMR}\left(\mathrm{DMSO}-d_{6}, 400 \mathrm{MHz}, \delta \mathrm{ppm}\right): 3.73\left(\mathrm{~s}, 3 \mathrm{H}, \mathrm{OCH}_{3}\right), 6.93(\mathrm{~d}, 2 \mathrm{H}, J$ $=9.0 \mathrm{~Hz}, \mathrm{ArH}), 7.12(\mathrm{t}, 1 \mathrm{H}, \mathrm{ArH}), 7.39(\mathrm{t}, 2 \mathrm{H}, \mathrm{ArH}), 7.41(\mathrm{~d}, 1 \mathrm{H}, J=4.8 \mathrm{~Hz}$, pyrimidine $), 7.59$ $(\mathrm{d}, 2 \mathrm{H}, J=9.0 \mathrm{~Hz}, \mathrm{ArH}), 7.73$ (d, 2H, $J=7.6 \mathrm{~Hz}, \mathrm{ArH}), 7.90$ (d, 2H, $J=8.7 \mathrm{~Hz}, \mathrm{ArH}), 8.20$ (d, $2 \mathrm{H}, J=8.7 \mathrm{~Hz}, \mathrm{ArH}), 8.74(\mathrm{~d}, 1 \mathrm{H}, J=4.8 \mathrm{~Hz}$, pyrimidine), $9.26(\mathrm{~s}, 1 \mathrm{H}, \mathrm{NH}), 10.03(\mathrm{~s}, 1 \mathrm{H}, \mathrm{NH})$. ${ }^{13} \mathrm{C}-\mathrm{NMR}$ (DMSO- $\left.d_{6}, 100 \mathrm{MHz}, \delta \mathrm{ppm}\right): 55.7\left(\mathrm{C}, \mathrm{OCH}_{3}\right), 87.6\left(\mathrm{C}, \mathrm{C}_{3}\right.$-pyrazolopyrimidine), 106.9 (C, $\mathrm{C}_{6}$-pyrazolopyrimidine), 114.4, 119.1, 120.5, 123.3, 129.4, 129.8, 131.0, 131.6 (14C, Ar), 133.7 (C, $\mathrm{C}_{3 \mathrm{a}}$-pyrazolopyrimidine $), 133.4,136.1,138.7$ (3C, $\left.\mathrm{Ar}\right), 145.2\left(\mathrm{C}, \mathrm{C}_{7^{-}}\right.$ pyrazolopyrimidine), 149.5 (C, Ar), 154.8 (C, $\mathrm{C}_{2}$-pyrazolopyrimidine), 157.1 (C, $\mathrm{C}_{5^{-}}$ pyrazolopyrimidine), $163.7(\mathrm{C}=\mathrm{O})$. MS $(\mathrm{m} / \mathrm{z}, \%)$ : $514\left(\mathrm{M}^{+}, 81.26\right)$. Anal. calcd. (\%) for $\mathrm{C}_{26} \mathrm{H}_{20} \mathrm{BrN}_{5} \mathrm{O}_{2}$ (514.37): C, 60.71; H, 3.92; N, 13.62. Found: $\mathrm{C}, 60.65 ; \mathrm{H}, 3.97 ; \mathrm{N}, 13.65 \%$.

7-(4-Fluorophenyl)-2-(4-methoxyphenylamino)-N-phenylpyrazolo[1,5-a]pyrimidine-3carboxamide (5f). Yellow crystals, m.p. $237-239{ }^{\circ} \mathrm{C}$, yield $(70 \%)$. IR $(\mathrm{KBr}) v_{\max } / \mathrm{cm}^{-1} 3343$ $(\mathrm{NH}), 1647(\mathrm{C}=\mathrm{O}) .{ }^{1} \mathrm{H}-\mathrm{NMR}\left(\mathrm{DMSO}-d_{6}, 400 \mathrm{MHz}, \delta \mathrm{ppm}\right): 3.73\left(\mathrm{~s}, 3 \mathrm{H}, \mathrm{OCH}_{3}\right), 6.91(\mathrm{~d}, 2 \mathrm{H}, J$ $=9.0 \mathrm{~Hz}, \mathrm{ArH}), 7.11(\mathrm{t}, 1 \mathrm{H}, \mathrm{ArH}), 7.37(\mathrm{~d}, 1 \mathrm{H}, J=4.9 \mathrm{~Hz}$, pyrimidine $), 7.39(\mathrm{~d}, 2 \mathrm{H}, J=7.6 \mathrm{~Hz}$, ArH), 7.52 (t, 2H, ArH), 7.58 (d, 2H, $J=9.0 \mathrm{~Hz}, \mathrm{ArH}), 7.71$ (d, 2H, $J=8.6 \mathrm{~Hz}, \mathrm{ArH}), 8.31$ (d, $2 \mathrm{H}, J=8.9 \mathrm{~Hz}, \mathrm{ArH}), 8.71(\mathrm{~d}, 1 \mathrm{H}, J=4.8 \mathrm{~Hz}$, pyrimidine), $9.23(\mathrm{~s}, 1 \mathrm{H}, \mathrm{NH}), 10.01(\mathrm{~s}, 1 \mathrm{H}, \mathrm{NH})$. ${ }^{13} \mathrm{C}-\mathrm{NMR}$ (DMSO- $\left.d_{6}, 100 \mathrm{MHz}, \delta \mathrm{ppm}\right): 55.2\left(\mathrm{C}, \mathrm{OCH}_{3}\right), 86.7$ (C, $\mathrm{C}_{3}$-pyrazolopyrimidine), 108.3 (C, $\mathrm{C}_{6}$-pyrazolopyrimidine), 114.3, 115.7, 115.9, 118.8, 119.4, 123.5, 126.4, 129.1 (14C, Ar), $\quad 132.4 \quad\left(\mathrm{C}, \quad \mathrm{C}_{3 \mathrm{a}}\right.$-pyrazolopyrimidine $), \quad 133.3, \quad 138.4 \quad(2 \mathrm{C}, \quad \mathrm{Ar}), \quad 145.0 \quad\left(\mathrm{C}, \mathrm{C}_{7^{-}}\right.$ pyrazolopyrimidine $), 147.1(\mathrm{C}, \mathrm{Ar}), 151.1 \quad\left(\mathrm{C}, \mathrm{C}_{2}\right.$-pyrazolopyrimidine $), 154.1 \quad\left(\mathrm{C}, \mathrm{C}_{5^{-}}\right.$ pyrazolopyrimidine), $156.6(\mathrm{C}, \mathrm{Ar}), 162.2(\mathrm{C}=\mathrm{O})$. $\mathrm{MS}(\mathrm{m} / \mathrm{z}, \%): 453\left(\mathrm{M}^{+}, 87.33\right)$. Anal. calcd. (\%) for $\mathrm{C}_{26} \mathrm{H}_{20} \mathrm{FN}_{5} \mathrm{O}_{2}$ (453.47): $\mathrm{C}, 68.86 ; \mathrm{H}, 4.45 ; \mathrm{N}, 15.44$. Found: $\mathrm{C}, 68.95 ; \mathrm{H}, 4.40 ; \mathrm{N}$, $15.50 \%$.

\section{RESULTS AND DISCUSSION}

\section{Characterization of the synthesized catalyst}

Figure 1 reveals the X-ray diffraction pattern of the synthesized copper-based glass-ceramic catalyst. The obtained pattern shows multiple sharp bands superimposed on a broad background spectrum. The background spectrum with attributed to the amorphous silicate network while the 
sharp bands were attributed to the precipitated crystalline phases during the annealing process. It was observed that the spectrum refers to a crystalline multiphase structure consists of at least three phases identified through the JCPDS library.

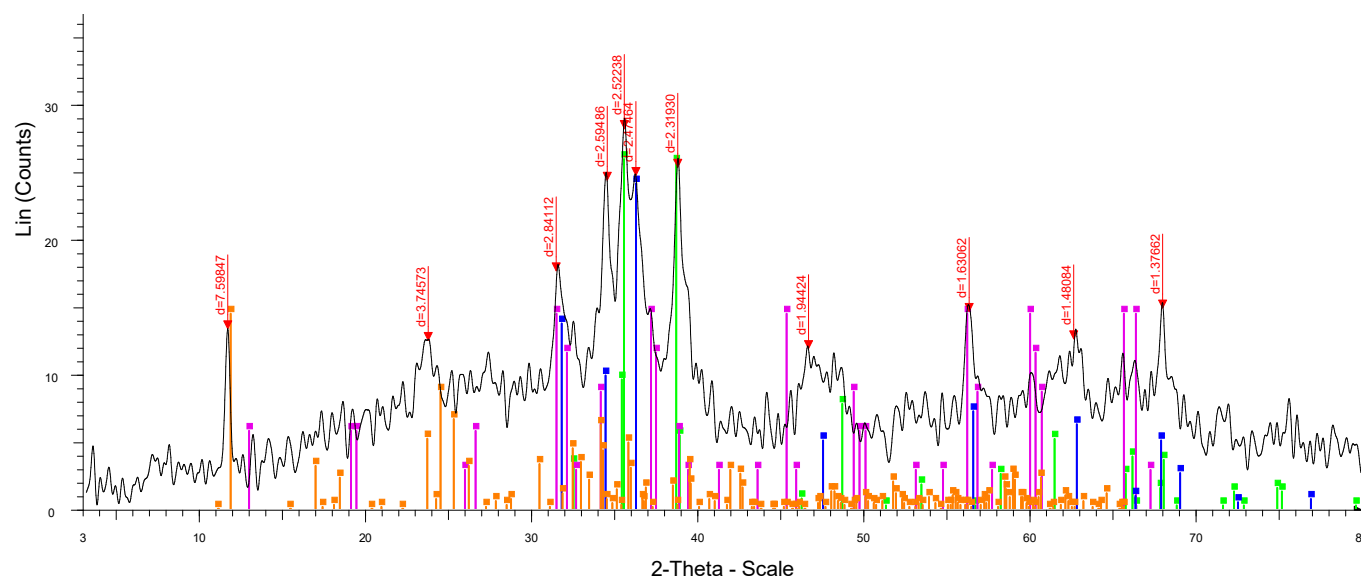

Figure 1. XRD pattern of the prepared catalyst.

Scanning electron microscopy images supported with EDAX (SEM/EDAX) shown in Figure 2 reveals the same result retained from the XRD pattern in addition to more information about the surface morphology and composition. A homogenous sample with normal elemental distribution without voids or clusters was shown in Figure 2a. Mapping image Figure $2 \mathrm{~b}$ supports the homogeneity of distribution while Figure 2c introduces the analysis of a presented component with an agreement with that used in the synthesis process.

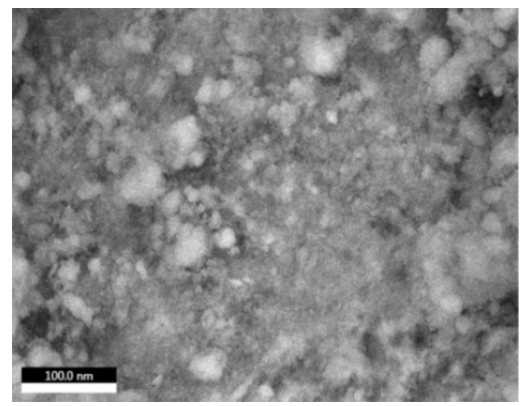

(a)

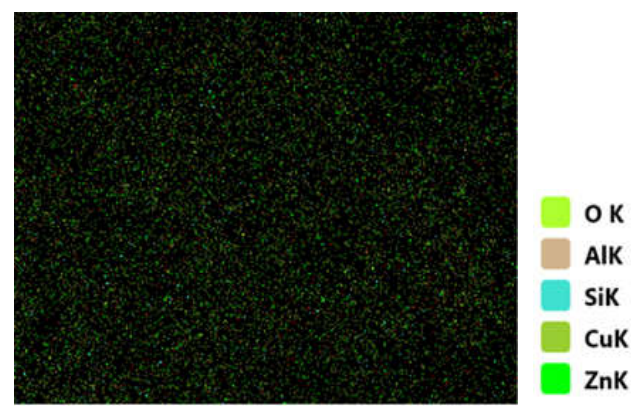

(b) 


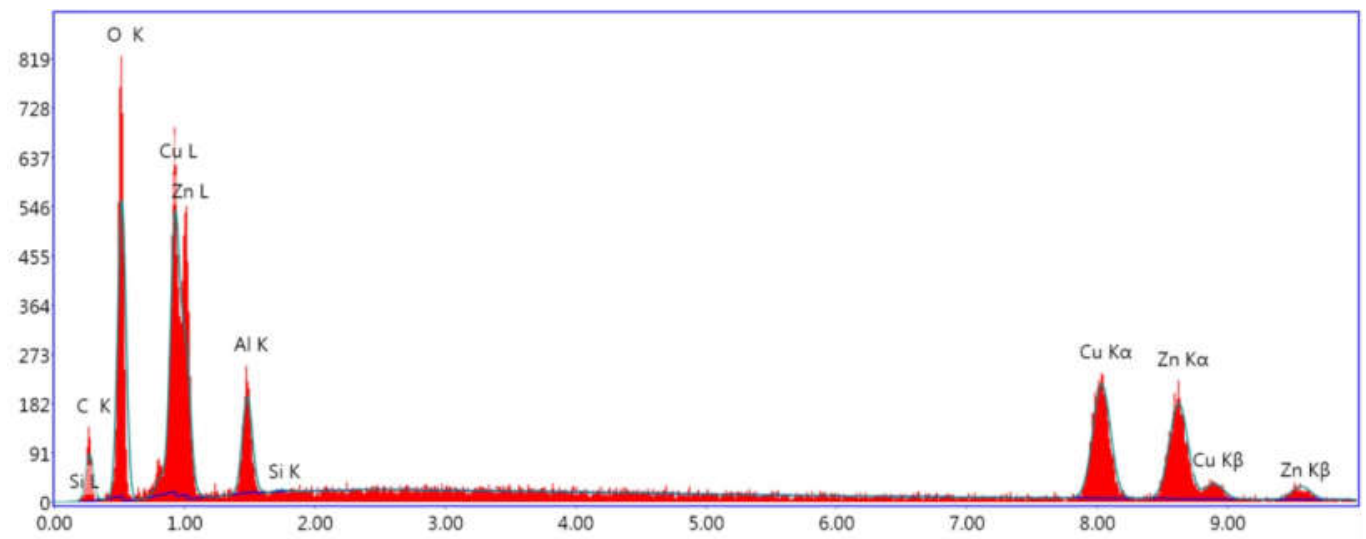

(c)

Figure 2. (a) SEM image of synthesized catalyst, (b) mapping, and (c) EDAX analysis.

Organic synthesis

The most common traditional method for the synthesis of 7-aryl-2-(phenylamino)pyrazolo[1,5a]pyrimidine-3-carboxamide 5a-f via the reaction of 5-amino-3-(4-methoxyphenylamino)- $\mathrm{N}$ phenyl-1H-pyrazole-4-carboxamide (1) with enaminones 2a-f in refluxed $\mathrm{CH}_{3} \mathrm{COOH}$ [34]. And towards, the aim of our research group for discovering new green methods for the synthesis of organic compounds [35-39]. The pyrazolo[1,5-a]pyrimidines 5a-f were prepared under solventfree conditions in the presence of green, cheap, and recyclable catalyst composed of glassceramic oxides powder. The obtained results explained that the suggested catalytic method gives more yield with green conditions shown in Scheme 1.
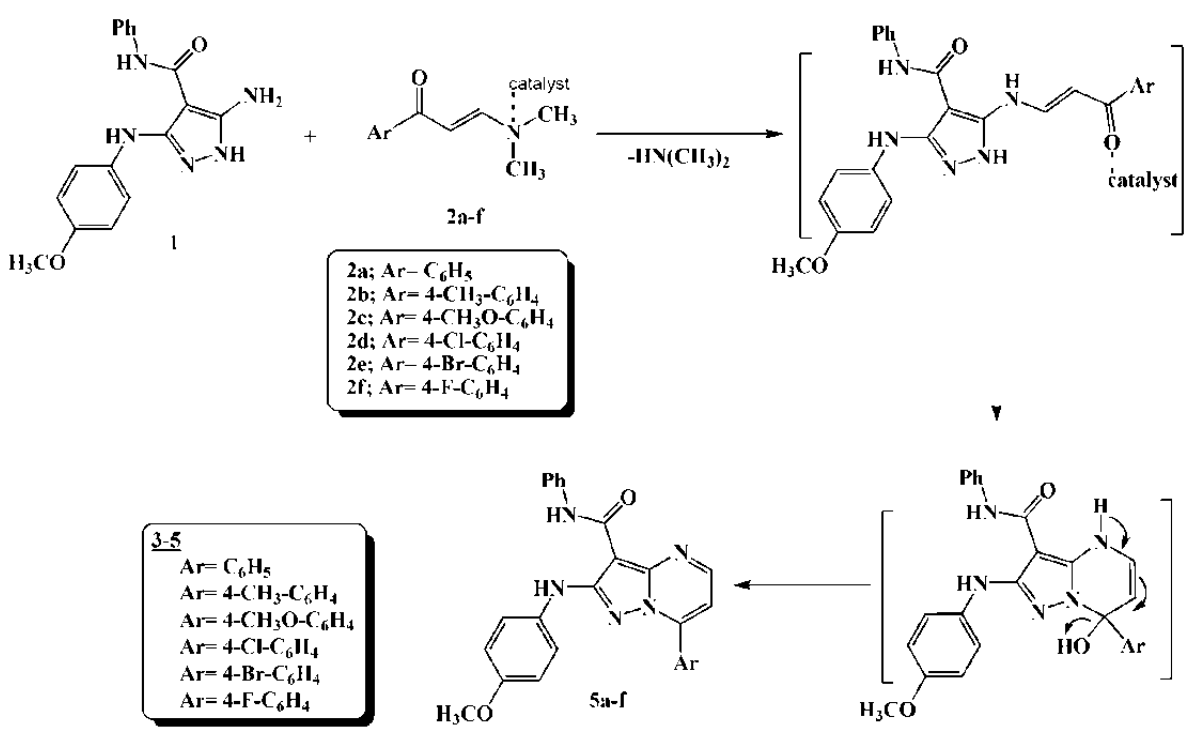

Scheme 1. Synthesis of compound 5a-f.

Bull. Chem. Soc. Ethiop. 2021, 35(1) 
The suggested mechanism was explained as one can see in Scheme 1. The enamine was activated by the catalyst through the coordinate bond with an amino group in enamine and converted it to quaternary amine form which considered as a good leaving group than tertiary amine and in the second step, catalyst plays an important role in the activation of enone group by increase the polarization of carbonyl group. In the final step the intermediate loss of water molecules and give the stable form pyrazolo[1,5- $a]$ pyrimidines $\mathbf{5 a - f .}$

Table 1. Synthesis of pyrazolopyrimidines 5a-f from 5-aminopyrazole and enaminones.

\begin{tabular}{|c|c|c|c|c|}
\hline \multicolumn{2}{|c|}{ Entry } & $\mathrm{Ar}$ & Yield (\%) & Time (h) \\
\hline 1 & $\mathbf{5 a}$ & $\mathrm{C}_{6} \mathrm{H}_{5}$ & 88 & 9 \\
\hline 2 & $\mathbf{5 b}$ & $4-\mathrm{CH}_{3}-\mathrm{C}_{6} \mathrm{H}_{4}-$ & 86 & 10 \\
\hline 3 & $\mathbf{5 c}$ & $4-\mathrm{CH}_{3} \mathrm{O}-\mathrm{C}_{6} \mathrm{H}_{4}-$ & 90 & 5 \\
\hline 4 & $\mathbf{5 d}$ & $4-\mathrm{Cl}-\mathrm{C}_{6} \mathrm{H}_{4-}$ & 93 & 6 \\
\hline 5 & $\mathbf{5 e}$ & $4-\mathrm{Br}-\mathrm{C}_{6} \mathrm{H}_{4-}$ & 91 & 8 \\
\hline 6 & $\mathbf{5 f}$ & $4-\mathrm{F}-\mathrm{C}_{6} \mathrm{H}_{4-}$ & 92 & 5 \\
\hline
\end{tabular}

From the data showed in Table 1, we noticed that when (Ar) substituted by the electronwithdrawing groups like the presence of halogens in para position reaction gives more yield and less reaction time. Besides, the presence of an electron releasing group in the aromatic ring at (Ar) gives lower yields and less reaction time.

\section{Molecular docking study}

The affinity and efficacy of the drug can be expected using molecular docking software. The binding between active sites in the COVID-19 main protease $\left(\mathrm{M}^{\mathrm{pro}}\right)$ as a targeting enzyme was docked by pyrazolopyrimidines. The result was obtained by comparison with hydroxychloroquine as a reference molecule. Hydroxychloroquine during the last few months considered a promised candidate drug for Covid-19. In this special corona virus pandemic, $\mathrm{M}^{\text {pro }}$ is considered as a valuable target and unique challenge. The active binding site is presented by Liu et al. [40] and in the extension of our research group [41-44] to discover new efficient ligand-receptor interaction.

The E-score (energy score in $\mathrm{kcal} / \mathrm{mol}$ ) is considered as one of the significant factors that explained the binding process between the ligand and enzyme. The molecular docking validation clarifies the interaction (E-score) between pyrazolopyrimidines 5a-f with a $\mathrm{M}^{\text {pro }}$ enzyme as $-6.93,-8.24,-6.93,-6.88,-7.63$, and -6.88 , respectively. The calculated data explained that the protease active site protein residues are Asn 142, Thr 45, Thr 26, Glu 166, His 41, Thr 25, Gln 192, Thr 190, Arg 188, Ala 191, Met 165, Leu 167, Leu 141, Asp 187, Thr 42, Pro 168, His 164, Gly 143, Ser 144, Cys 145, Gln 189, Met 49, Leu 27, Ser 46, Cys 44. Figures $3 \mathrm{a}$ and $3 \mathrm{~b}$ showed the $2 \mathrm{D}$ and $3 \mathrm{D}$ interaction diagrams with $\mathrm{M}^{\text {pro }}$. 


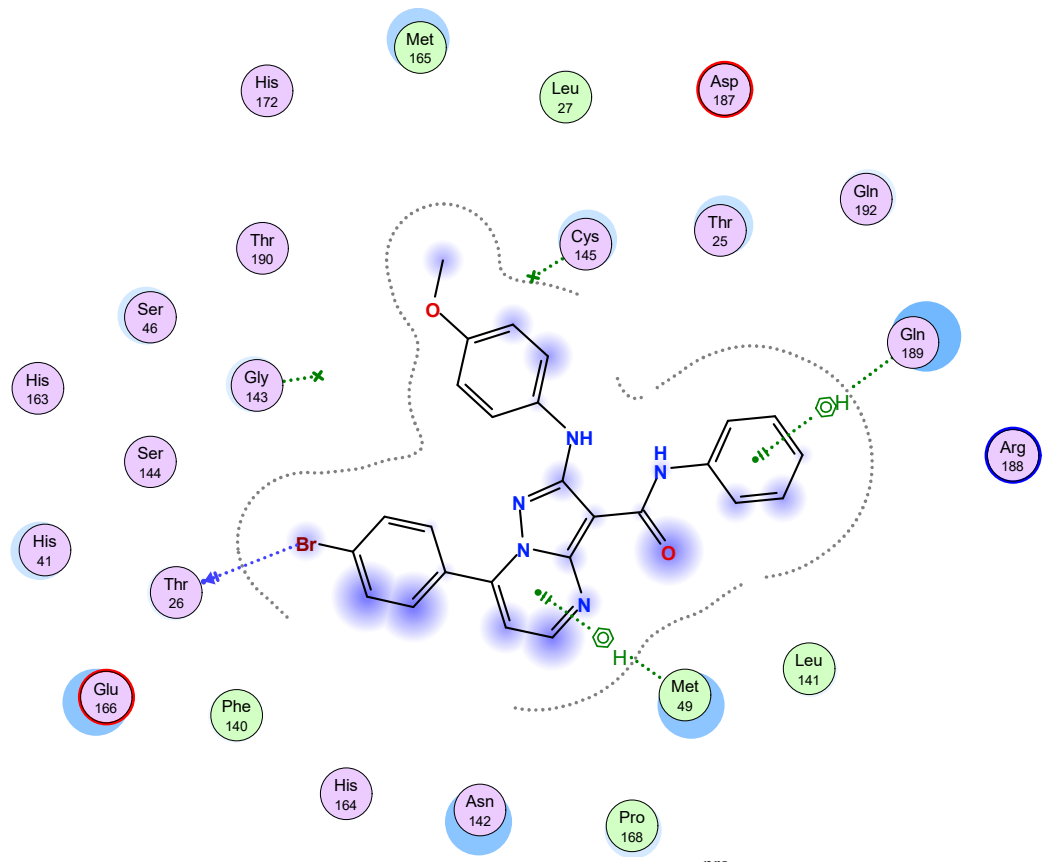

Figure 3a. 2D of the drug ligand interaction between $5 \mathbf{e}$ and $\mathrm{M}^{\text {pro }}$ enzyme active side.

The drug-ligand electrostatic force-distance measurements

The measured distance also reflects good drug ligand interaction. For example, we presented the drug-ligand electrostatic force-distance measurements for compound $5 \mathbf{e}$ the hydrogen bond distance between Thr 26 and the bromo group equal 3.31 ; the $\pi-\pi$ stacking also presented between Gln 189 and Met 49 with the two aromatic rings. This compound presented 7 intramolecular forces that presented the high drug ligand interaction.

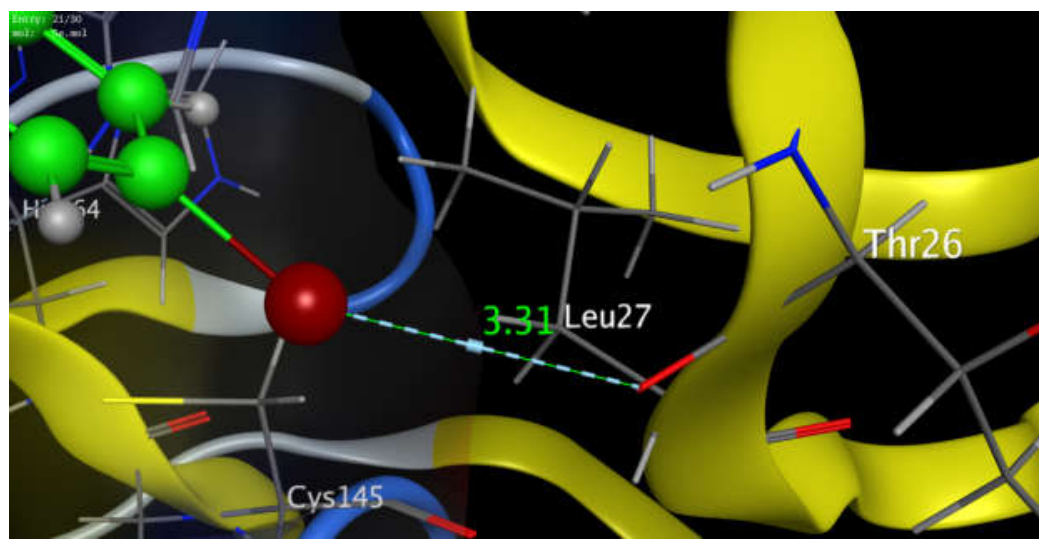

Figure $3 b$. The distance measurements in $3 \mathrm{D}$ interaction diagrams of $\mathbf{5 e}$ with $\mathrm{M}^{\text {pro }}$ enzyme binding active side. 


\section{CONCLUSION}

In conclusion, the oxide glass-ceramic catalyst powder was prepared and characterized. The prepared catalyst was applied successfully in the synthesis of pyrazolo[1,5-a]pyrimidines 5a-f via the reaction of 5-amino-1H-pyrazole-4-carboxamide $\mathbf{1}$ with enaminones $\mathbf{2 a - f}$ under solventfree condition. The molecular docking study proved that the prepared compounds presented good affinity with the COVID-19 main protease $\left(\mathrm{M}^{\mathrm{Pro}}\right)$.

\section{ACKNOWLEDGEMENTS}

The authors wish to express their thanks to the National Research Centre for the facilities provided.

\section{REFERENCES}

1. Volla, C.M.; Atodiresei, I.; Rueping, M. Catalytic C-C bond-forming multi-component cascade or domino reactions: Pushing the boundaries of complexity in asymmetric organocatalysis. Chem. Rev. 2014, 114, 2390-2431.

2. Sudarsanam, P.; Peeters, E.; Makshina, E.V.; Parvulescu, V.I.; Sels, B.F. Advances in porous and nanoscale catalysts for viable biomass conversion. Chem. Soc. Rev. 2019, 48, 2366-2421.

3. Twigg, M.V. Catalyst Handbook, 2nd ed., Routledge: England; 2018.

4. Ratnasamy, C.; Wagner, J.P. Water gas shift catalysis. Catal. Rev. 2009, 51, 325-440.

5. Özcan, M.; Vallittu, P.K. Effect of surface conditioning methods on the bond strength of luting cement to ceramics. Dent. Mater. 2003, 19, 725-731.

6. Hassan, A.S.; Hafez, T.S.; Osman, S.A.; Ali, M.M. Synthesis and in vitro cytotoxic activity of novel pyrazolo[1,5-a]pyrimidines and related Schiff bases. Turk. J. Chem. 2015, 39, 1102-1113.

7. Peytam, F.; Adib, M.; Shourgeshty, R.; firoozpour, L.; R.-Jazi, M.; Jahani, M.; Moghimi, S.; Divsalar, K.; faramarzi, M.A.; Mojtabavi, S.; Safari, F.; Mahdavi, M.; foroumadi, A. An efcient and targeted synthetic approach towards new highly substituted 6-aminopyrazolo[1,5- $a$ ]pyrimidines with $\alpha$-glucosidase inhibitory activity. Sci. Rep. 2020, 10, 2595.

8. Hosny, M.A.; Zaki, Y.H.; Mokbel, W.A.; Abdelhamid, A.O. Synthesis, characterization, antimicrobial activity and anticancer of some new pyrazolo[1,5-a]pyrimidines and pyrazolo[5,1-c]1,2,4-triazines. Med. Chem. 2020, 16, 750-760.

9. Kaping, S.; Boiss, I.; Singha, L.I.; Helissey, P.; Vishwakarma, J.N. A facile, regioselective synthesis of novel 3-( $N$-phenylcarboxamide)pyrazolo[1,5-a]pyrimidine analogs in the presence of $\mathrm{KHSO}_{4}$ in aqueous media assisted by ultrasound and their antibacterial activities. Mol. Divers. 2016, 20, 379-390.

10. Shen, J.; Deng, X.; Sun, R.; Tavallaie, M.S.; Wang, J.; Cai, Q.; Lam, C.; Lei, S.; Fu, L.; Jiang, F. Structural optimization of pyrazolo[1,5-a]pyrimidine derivatives as potent and highly selective DPP-4 inhibitors. Eur. J. Med. Chem. 2020, 208, 112850.

11. Li, G.; Wang, Y.; Li, L.; Ren, Y.; Deng, X.; Liu, J.; Wang, W.; Luo, M.; Liu, S.; Chen, J. Design, synthesis, and bioevaluation of pyrazolo[1,5-a]pyrimidine derivatives as tubulin polymerization inhibitors targeting the colchicine binding site with potent anticancer activities. Eur. J. Med. Chem. 2020, 202, 112519.

12. Husseiny, E.M. Synthesis, cytotoxicity of some pyrazoles and pyrazolo[1,5-a]pyrimidines bearing benzothiazole moiety and investigation of their mechanism of action. Bioorg. Chem. 2020, 102, 104053. 
13. Fahim, A.M.; Farag, A.M. Synthesis, antimicrobial evaluation, molecular docking and theoretical calculations of novel pyrazolo[1,5-a]pyrimidine derivatives. J. Mol. Struct. 2020, $1199,127025$.

14. Hwang, J.Y.; Windisch, M.P.; Jo, S.; Kim, K.; Kong, S.; Kim, H.C.; Kim, S.; Kim, H.; Lee, M.E.; Kim, Y.; Choi, J.; Park, D.-S.; Park, E.; Kwon, J.; Nam, J.; Ahn, S.; Cechetto, J.; Kim, J.; Liuzzi, M.; No, Z.; Lee, J. Discovery and characterization of a novel 7aminopyrazolo[1,5-a]pyrimidine analog as a potent hepatitis $\mathrm{C}$ virus inhibitor. Bioorg. Med. Chem. Lett. 2012, 22, 7297-7301.

15. Jiang, J.-K.; Huang, X.; Shamim, K.; Patel, P.R.; Lee, A.; Wang, A.Q.; Nguyen, K.; Tawa, G.; Cuny, G.D.; Yu, P.B.; Zheng, W.; Xu, X.; Sanderson, P.; Huang, W. Discovery of 3-(4sulfamoylnaphthyl)pyrazolo[1,5-a]pyrimidines as potentand selective ALK2 inhibitors. Bioorg. Med. Chem. Lett. 2018, 28, 3356-3362.

16. Attia, M.H.; Elrazaz, E.Z.; El-Emam, S.Z.; Taher, A.T.; Abdel-Aziz, H.A.; Abouzid, K.A.M. Synthesis and in-vitro anti-proliferative evaluation of some pyrazolo[1,5a]pyrimidines as novel larotrectinib analogs. Bioorg. Chem. 2020, 94, 103458.

17. Singleton, J.D.; Dass, R.; Neubert, N.R.; Smith, R.M.; Webber, Z.; Hansen, M.D.H.; Peterson, M.A. Synthesis and biological evaluation of novel pyrazolo[1,5-a]pyrimidines: Discovery of a selective inhibitor of JAK1 JH2 pseudokinase and VPS34. Bioorg. Med. Chem. Lett. 2020, 30, 126813.

18. Khatab, T.K.; Abdelghany, A.M.; Kandil, E.M.; Elsefy, D.E.; El-Mekabaty, A. Hydroxyapatite $/ \mathrm{ZnCl}_{2}$ nano-flakes: An efficient catalyst for the synthesis of 2-arylbenzothiazoles with molecular docking and anti-oxidant evaluation. Biointerface Res. Appl. Chem. 2020, 10, 5182-5187.

19. Abdelghany, A.M.; Menazea, A.A.; Abd-El-Maksoud, M.A.; Khatab, T.K. Pulsed laser ablated zeolite nanoparticles: A novel nano-catalyst for the synthesis of 1,8-dioxooctahydroxanthene and $N$-aryl-1,8-dioxodecahydroacridine with molecular docking validation. Appl. Organometal. Chem. 2020, 34, e5250.

20. Sroor, F.M.; Khatab, T.K.; Basyouni, W.M.; El-Bayouki, K.A.M. Synthesis and molecular docking studies of some new thiosemicarbazone derivatives as HCV polymeraseinhibitors. Synth. Commun. 2019, 49, 1444-1456.

21. Khatab, T.K.; Abdelghany, A.M.; Soliman, H.A. $\mathrm{V}_{2} \mathrm{O}_{5}$ based quadruple nano-perovskite as a new catalyst for the synthesis of bis and tetrakis heterocyclic compounds. Appl. Organometal. Chem. 2019, 33, e4783.

22. Elsherif, M.A.; Hassan, A.S.; Moustafa, G.O.; Awad, H.M.; Morsy, N.M. Antimicrobial evaluation and molecular properties prediction of pyrazolines incorporating benzofuran and pyrazole moieties. J. Appl. Pharm. Sci. 2020, 10, 37-43.

23. Hassan, A.S.; Askar, A.A.; Nossier, E.S.; Naglah, A.M.; Moustafa, G.O.; Al-Omar, M.A. Antibacterial evaluation, in silico characters and molecular docking of Schiff bases derived from 5-aminopyrazoles. Molecules 2019, 24, 3130.

24. Hassan, A.S.; Moustafa, G.O.; Askar, A.A.; Naglah, A.M.; Al-Omar, M.A. Synthesis and antibacterial evaluation of fused pyrazoles and Schiff bases. Synth. Commun. 2018, 48, 2761-2772.

25. Magd-El-Din, A.A.; Mousa, H.A.; Labib, A.A.; Hassan, A.S.; Abd El-All, A.S.; Ali, M.M.; El-Rashedy, A.A.; El-Desoky, A.H. Benzimidazole-Schiff bases and their complexes: Synthesis, anticancer activity and molecular modeling as Aurora kinase inhibitor. $Z$. Naturforsch. C 2018, 73, 465-478.

26. Hassan, A.S.; Hafez, T.S. Antimicrobial activities of ferrocenyl complexes: A review. $J$. App. Pharm. Sci. 2018, 8, 156-165.

27. Hassan, A.S.; Awad, H.M.; Magd-El-Din, A.A.; Hafez, T.S. Synthesis and in vitro antitumor evaluation of novel Schiff bases. Med. Chem. Res. 2018, 27, 915-927. 
28. Hassan, A.S.; Moustafa, G.O.; Awad, H.M. Synthesis and in vitro anticancer activity of pyrazolo[1,5-a]pyrimidines and pyrazolo[3,4-d][1,2,3]triazines. Synth. Commun. 2017, 47, 1963-1972.

29. Hassan, A.S.; Hafez, T.S.; Ali, M.M.; Khatab, T.K. Design, synthesis and cytotoxic activity of some new pyrazolines bearing benzofuran and pyrazole moieties. Res. J. Pharm. Biol. Chem. Sci. 2016, 7, 417-429.

30. Abd El-All, A.S.; Hassan, A.S.; Osman, S.A.; Yosef, H.A.A.; Abdel-Hady, W.H.; ElHashash, M.A.; Atta-Allah, S.R.; Ali, M.M.; El Rashedy, A.A. Synthesis, characterization and biological evaluation of new fused triazine derivatives based on 6-methyl-3-thioxo1,2,4-triazin-5-one. Acta Pol. Pharm. 2016, 73, 79-92.

31. Hassan, A.S.; Osman, S.A.; Hafez, T.S. 5-Phenyl-2-furaldehyde: Synthesis, reactions and biological activities. Egypt. J. Chem. 2015, 58, 113-139.

32. Hassan, A.S.; Askar, A.A.; Naglah, A.M.; Almehizia, A.A.; Ragab, A. Discovery of new Schiff bases tethered pyrazole moiety: Design, synthesis, biological evaluation, and molecular docking study as dual targeting DHFR/DNA Gyrase inhibitors with immune modulatory activity. Molecules 2020, 25, 2593.

33. Al-Wasidi, A.S.; Hassan, A.S.; Naglah, A.M. In vitro cytotoxicity and druglikeness of pyrazolines and pyridines bearing benzofuran moiety. J. App. Pharm. Sci. 2020, 10, 142148.

34. El-Naggar, M.; Hassan, A.S.; Awad, H.M.; Mady, M.F. Design, synthesis and antitumor evaluation of novel pyrazolopyrimidines and pyrazoloquinazolines. Molecules 2018, 23, 1249.

35. Soliman, H.A.; Khatab, T.K.; Abdel-Megeid, F.M.E. Utilization of bromine azide to access vicinal-azidobromides from arylidenemalononitrile. Chin. Chem. Lett. 2016, 27, 1515-1518.

36. Soliman, H.A.; Khtab, T.K. Efficient heterogeneous catalytic one-pot, three component synthesis of $\gamma$-hydroxy- $\beta$-ketoamides. Egypt. J. Chem. 2014, 57, 129-142.

37. Khatab, T.K.; El-Bayouki, K.A.M.; Basyouni, W.M.; El-Basyoni, F.A.; Ali, M.M.; Abbas, S.Y.; Mostafa, E.A. Sulfamicacid as an efficient, cost-effective, eco-friendly and recyclable solid acid catalyst for the synthesis of a novel series of 2,3-dihydroquinazolin-4(1H)-ones and antitumor evaluation. Res. J. Pharm. Biol. Chem. Sci. 2015, 6, 281.

38. Khatab, T.K.; Elmorsy, S.S.; Badawy, D.S. Evaluation of $\mathrm{TCS} / \mathrm{ZnCl}_{2}$ with acetic anhydride as an acetylating reagent for methylene ketones. Phosphorus Sulfur Silicon Relat. Elem.2005, 180, 109-116.

39. Khatab, T.K.; Hassan, A.S.; Hafez, T.S. $\mathrm{V}_{2} \mathrm{O}_{5} / \mathrm{SiO}_{2}$ as an efficient catalyst in the synthesis of 5-aminopyrazole derivatives under solvent free condition. Bull. Chem. Soc. Ethiop. 2019, $33,135-142$.

40. Jin, Z.; Zhao, Y.; Sun, Y.; Zhang, B.; Wang, H.; Wu, Y.; Zhu, Y.; Zhu, C.; Hu, T.; Du, X.; Duan, Y.; Yu, J.; Yang, X.; Yang, X.; Yang, K.; Liu, X.; Guddat, L.W.; Xiao, G.; Zhang, L.; Yang, H.; Rao, Z. Structural basis for the inhibition of SARS-CoV-2 main protease by antineoplastic drug carmofur. Nat. Struct. Mol. Biol. 2020, 27, 529-532.

41. Naglah, A.M.; Askar, A.A.; Hassan, A.S.; Khatab, T.K.; Al-Omar, M.A.; Bhat, M.A. Biological evaluation and molecular docking with in silico physicochemical, pharmacokinetic and toxicity prediction of pyrazolo[1,5-a]pyrimidines. Molecules 2020, 25, 1431.

42. Khatab, T.K.; Mubarak, A.Y.; Soliman, H.A. Design and synthesis pairing between xanthene and tetrazole in pentacyclic system using tetrachlorosilane with aurora kinase inhibitor validation. J. Heterocycl. Chem. 2017, 54, 2463-2470.

43. Khatab, T.K.; El-Bayouki, K.A.M., Basyouni, W.M.; Sroor, F.M.A. Efficient synthesis of biopertinentdihydropyrimidine (thi) one derivatives via three-component one-pot synthesis catalyzed by tetrachlorosilane. Egypt. J. Chem. 2013, 56, 291-305. 
44. Badawy, D.S.; Abdel-Galil, E.; Kandeel, E.M.; Basyouni, W.M.; El-Bayouki, K.A.M.; Khatab, T.K. Tetrachlorosilane-zinc chloride as a new potent binary reagent for one-pot, three-component synthesis of Mannich-type products. Phosphorus Sulfur Silicon Relat. Elem. 2009, 184, 2799-2812. 